\title{
Tetrahydroabietic Acid, a Reduced Abietic Acid, Inhibits the Production of Inflammatory Mediators in RAW264.7 Macrophages Activated with Lipopolysaccharide
}

\author{
Nam-Ho Kim¹, Yong Son ${ }^{2}$, Sun-Oh Jeong 3 , Jong Moon Hur ${ }^{3}$, Han Soo Bang ${ }^{4}$, Ki-Nam Lee \\ Eun-Cheol Kim ${ }^{5}$, Hun-Taeg Chung ${ }^{6}$ and Hyun-Ock Pae ${ }^{3, *}$ \\ ${ }^{1}$ Department of Cardiovascular Medicine, Wonkwang University Hospital, Iksan 570-749, Republic of Korea \\ ${ }^{2}$ Department of Anesthesiology and Pain Medicine, Wonkwang University School of Medicine, \\ Iksan 570-749, Republic of Korea \\ ${ }^{3}$ Department of Microbiology and Immunology, Wonkwang University School of Medicine, \\ 344-2 Shinyong-dong, Iksan 570-749, Republic of Korea \\ ${ }^{4}$ Professional Graduate School of Oriental Medicine, Wonkwang University, Iksan 570-749, Republic of Korea \\ ${ }^{5}$ Department of Oral and Maxillofacial Pathology, College of Dentistry, Wonkwang University, \\ Iksan 570-749, Republic of Korea \\ ${ }^{6}$ Department of Biological Science, University of Ulsan, Ulsan 680-749, Republic of Korea
}

Received 13 July, 2009; Accepted 15 October, 2009

\begin{abstract}
Summary Abietic acid (AA), the main component of the rosin fraction of oleoresin synthesized by conifer species, has been reported to have anti-inflammatory effects. AA is a weak contact allergen; however, compounds resulting from its oxidation by air elicit stronger allergic response. Hydrogenation of the conjugated double bonds of $\mathrm{AA}$, as in tetrahydroabietic acid (THAA), decreases its susceptibility to air oxidation and would thus reduce the allergenicity of AA. The aim of this study was to investigate whether THAA could exert anti-inflammatory effects to the same extent as AA in RAW264.7 macrophages activated with the endotoxin lipopolysaccharide (LPS). THAA and AA inhibited the production of nitric oxide (NO) and prostaglandin $E_{2}$ by suppressing the expression of inducible NO synthase and cyclooxygenase-2, respectively, in LPS-activated RAW264.7 macrophages. They also inhibited

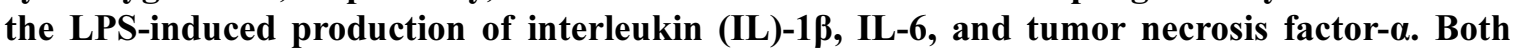
THAA and AA prevented the LPS-induced nuclear translocation of the nuclear factor- $\mathrm{K} B / \mathrm{p} 65$ subunit, suggesting that THAA may inhibit the production of pro-inflammatory mediators through the same mechanism as AA. In comparison, the anti-inflammatory effects of THAA and AA were almost identical, indicating that THAA retains the anti-inflammatory activity of AA at least in LPS-activated RAW264.7 macrophages.
\end{abstract}

Key Words: abietic acid, tetrahydroabietic acid, inducible nitric oxide synthase, cyclooxygenase-2, nuclear factor- $\kappa B$

*To whom correspondence should be addressed.

Tel: +82-63-850-6925 Fax: +82-63-842-6925

E-mail: hopae@wku.ac.kr
Introduction

Inflammation is a complex process regulated by a variety of immune cells and effector molecules. Among the immune cells, activated macrophages play key roles in the initiation and amplification of inflammation in a variety of inflammatory diseases via the excess production of pro-inflammatory 
cytokines, including tumor necrosis factor- $\alpha$ (TNF- $\alpha$ ), interleukin-1 $\beta$ (IL-1 $\beta$ ), and IL-6, and also the prolonged expression of inducible pro-inflammatory enzymes, including inducible nitric oxide synthase (iNOS) and cyclooxygenase2 (COX-2) [1]. The iNOS and COX-2, once expressed in activated macrophages, can synthesize large amount of nitric oxide (NO) and prostaglandin $\mathrm{E}_{2}\left(\mathrm{PGE}_{2}\right)$, respectively, for a long period [2]. Lipopolysaccharide (LPS), a principal component of the outer membrane of Gram-negative bacteria, activates macrophages to produce these inflammatory mediators mainly through activation of nuclear factor (NF)- $\kappa \mathrm{B}$ [3]. The transcription factor, $N F-\kappa B$, exists mainly as a heterodimer consisting of subunits of the Rel family p50 and p65, which are normally sequestered in the cytosol as an inactive complex due to binding with inhibitor proteins in unstimulated cells [4]. The activation of NF- $\kappa B$ by LPS in macrophages results in phosphorylation, ubiquitination, and proteasome-mediated degradation of the inhibitory proteins, followed by the translocation of p50/p65 heterodimer to the nucleus and the induction of transcriptions of proinflammatory mediators through binding to their binding sites in the promoter regions of target genes [5]. There are many studies demonstrating that naturally occurring phytochemicals, such as terpenoids and flavonoids, can inhibit the production of macrophage-mediated pro-inflammatory mediators via inhibition of $\mathrm{NF}-\kappa \mathrm{B}$ activation (reviewed in Ref. [6] and see references therein), and the compounds thus are considered to have anti-inflammatory effects.

Terpenoids, which are present in many dietary and herbal plants [7], have been regarded as valuable medicinal and food components that may regulate many cellular functions. Abietic acid (AA), a tricyclic diterpene carboxylic acid (chemical structure shown in Fig. 1), is the main component of the rosin fraction of oleoresin synthesized by conifer species, such as grand fir (Abies grandis) and lodgepole pine (Pinus contorta) [8]. AA is a weak contact allergen; however, compounds resulting from its oxidation by air elicit stronger allergic response [9]. Hydrogenation of the conjugated double bonds of AA, as in tetrahydroabietic acid (THAA; chemical structure shown in Fig. 1), decreases its

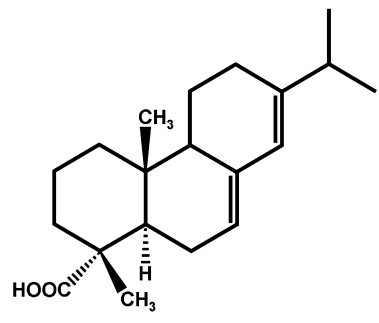

Abietic acid

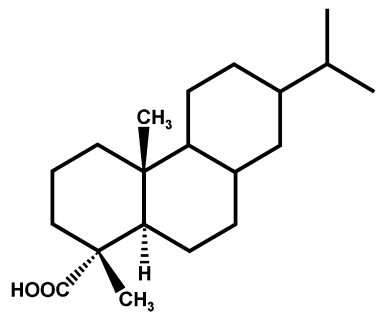

Tetrahydroabietic acid
Fig. 1. Chemical structures of abietic acid and tetrahydroabietic acid. susceptibility to air oxidation [10] and would thus reduce the allergenicity of AA.

Studies on the pharmacological activities of AA have demonstrated that AA suppresses the expression of proinflammatory enzymes and inhibits the production of proinflammatory cytokines in LPS-activated macrophages [11, 12]. However, whether THAA, even with its decreased allergenicity, could also exert the anti-inflammatory effects similar to those of AA remains to be investigated. The present study, therefore, was undertaken to evaluate the antiinflammatory effects of THAA in RAW264.7 macrophages and to compare its anti-inflammatory effects with those of its original form, AA.

\section{Materials and Methods}

\section{Chemicals and reagents}

AA and THAA were purchased from Wako Pure Chemical Industries (Osaka, Japan). Dulbecco's modified Eagle's medium (DMEM), LPS (Escherichia coli 055:B5), and 3(4,5-dimethyl-2-thiazolyl)-2,5-diphenyltetrazolium bromide (MTT) were purchased from Sigma-Aldrich (St. Louis, MO). Antibodies against iNOS, COX-2, p65, phospho (p)$\mathrm{I} \kappa \mathrm{B}, \mathrm{I} \kappa \mathrm{B} \alpha$, and $\beta$-actin were obtained from Santa Cruz Biotechnology (Santa Cruz, CA). The Enzyme-linked immunosorbent assay (ELISA) kits for PGE2, TNF- $\alpha$, IL-1 $\beta$, and IL-6 were purchased from R\&D Systems (Minneapolis, $\mathrm{MN})$.

\section{Cell culture}

RAW264.7 macrophages were obtained from the American Type Culture Collection (Manassas, VA). The cells were cultured in DMEM supplemented with $2 \mathrm{mM}$ glutamine, antibiotics $(100 \mathrm{U} / \mathrm{ml}$ of penicillin $\mathrm{A}$ and $100 \mathrm{U} / \mathrm{ml}$ of streptomycin) and 10\% heat-inactivated fetal bovine serum (Gibco/BRL, Rockville, MD) and maintained at $37^{\circ} \mathrm{C}$ in a humidified incubator containing $5 \% \mathrm{CO}_{2}$.

\section{Cell viability assay}

Cell viability was determined by a modified MTT reduction assay. MTT is a pale yellow substance that is reduced by living cells to yield a dark blue formazan product. This process requires active mitochondria, and even fresh dead cells do not reduce significant amounts of MTT. RAW264.7 macrophages were cultured in a 96-well flat-bottom plate at concentration of $5 \times 10^{5}$ cells $/ \mathrm{ml}$. After $12 \mathrm{~h}$ of preconditioning, the cells were treated with various concentrations of THAA or AA for $24 \mathrm{~h}$. Thereafter, culture medium was aspirated and $100 \mu \mathrm{l}$ of MTT dye $(1 \mathrm{mg} / \mathrm{ml}$ in phosphatebuffered saline) was added; the cultures were incubated for $4 \mathrm{~h}$ at $37^{\circ} \mathrm{C}$. The formazan crystals produced through dye reduction by viable cells were dissolved using acidified isopropanol $(0.1 \mathrm{~N} \mathrm{HCl})$. Index of cell viability was calcu- 
lated by measuring the optical density of color produced by MTT dye reduction at $570 \mathrm{~nm}$.

\section{Nitrite assay}

The nitrite concentration in the medium was measured as an indicator of NO production according to the Griess reaction. One hundred microliters of each supernatant was mixed with the same volume of Griess reagent (1\% sulfanilamide in $5 \%$ phosphoric acid and $0.1 \%$ naphthylethylenediamine dihydrochloride in water); absorbance of the mixture at $550 \mathrm{~nm}$ was determined with an ELISA plate reader.

\section{$P G E_{2}$ assay}

RAW264.7 macrophages were cultured in 24-well plates and were incubated with LPS in the presence or absence of AA or THAA for $24 \mathrm{~h}$. One hundred microliters of supernatant of culture medium was collected for the determination of $\mathrm{PGE}_{2}$ concentrations by ELISA.

\section{Western blot analysis}

RAW264.7 macrophages were incubated with or without reagents. They were harvested, washed ice-cold phosphatebuffered saline (PBS) and kept on ice for $1 \mathrm{~min}$. The suspension was mixed with buffer A (10 mM HEPES, pH 7.5, $10 \mathrm{mM} \mathrm{KCl}, 0.1 \mathrm{mM}$ EGTA, $0.1 \mathrm{mM}$ EDTA, $1 \mathrm{mM}$ DTT, $0.5 \mathrm{mM}$ PMSF, $5 \mu \mathrm{g} / \mathrm{ml}$ aprotinin, $5 \mu \mathrm{g} / \mathrm{ml}$ pepstatin, and $10 \mu \mathrm{g} / \mathrm{ml}$ leupeptin) and lysed by three freeze-thaw cycles. Cytosolic fraction was obtained by centrifugation at $12,000 \times \mathrm{g}$ for $20 \mathrm{~min}$ at $4^{\circ} \mathrm{C}$. The pellets were re-suspended in buffer $\mathrm{C}$ (20 mM HEPES, pH 7.5, $0.4 \mathrm{M} \mathrm{NaCl}, 1 \mathrm{mM}$ EGTA, $1 \mathrm{mM}$ EDTA, $1 \mathrm{mM}$ DTT, $1 \mathrm{mM}$ PMSF, $5 \mu \mathrm{g} / \mathrm{ml}$ aprotinin, $5 \mu \mathrm{g} / \mathrm{ml}$ pepstatin, and $10 \mu \mathrm{g} / \mathrm{ml}$ leupeptin) on ice for $40 \mathrm{~min}$ and centrifuged at $14,000 \times \mathrm{g}$ for $20 \mathrm{~min}$ at $4^{\circ} \mathrm{C}$. The resulting supernatant was used as soluble nuclear fraction. Protein content was determined with BCA protein assay reagent (Pierce, Rockford, IL). Total cellular or nuclear fractions were separated on 10\% SDS-polyacrylamide gels, and transferred to the nitrocellulose membranes (Amersham Biosciences, Inc., Piscataway, NJ). The membrane was then blocked in blocking buffer containing $20 \mathrm{mM}$ sodium phosphate buffer, pH 7.6, $150 \mathrm{mM} \mathrm{NaCl}, 0.1 \%$ Tween 20, and $5 \%$ nonfat dry milk for $1 \mathrm{~h}$ at room temperature. Thereafter, the membrane was incubated with antibodies against iNOS (1:1000 dilution), COX-2 (1:1000 dilution), p65 (1:500 dilution), or $\beta$-actin (1:1000 dilution) at $4{ }^{\circ} \mathrm{C}$ overnight. The membrane was then washed four times with PBS-Tween 20 buffer and further incubated with secondary antibody for $1 \mathrm{~h}$ at room temperature. Specific bands were detected using enhanced chemiluminescence detection system (Amersham Biosciences), and the membrane was exposed to X-ray film.

\section{Cytokine assay}

RAW264.7 macrophages $\left(5 \times 10^{5}\right.$ cells $\left./ \mathrm{ml}\right)$ were plated onto 24-well plates and pretreated with 5, 10, 20, or $40 \mu \mathrm{g} / \mathrm{ml}$ of THAA or AA for $1 \mathrm{~h}$ and then stimulated with $1 \mu \mathrm{g} / \mathrm{ml}$ of LPS for $24 \mathrm{~h}$. The cell supernatants were collected and stored at $-20^{\circ} \mathrm{C}$ until they were assayed for cytokines. The concentrations of TNF- $\alpha$, IL-1 $\beta$, and IL- 6 in the cell supernatants were measured by ELISA kits according to the manufacturer's instructions.

\section{$N F-\kappa B / p 65$ transcription factor assay}

NF- $\kappa$ B activity was determined with the TransAM ${ }^{\circledR}$ transcription factor assay kit (Active Motif, Carlsbad, CA) following the manufacturer's protocol. In brief, $5 \mu \mathrm{g}$ of nuclear extract was added to each well of a 96-well plate precoated with $\mathrm{NF}-\kappa \mathrm{B}$ consensus binding oligonucleotide (5'-GGGACTTTCC-3'). After 1 h of incubation with smooth agitation, wells were washed three times with washing buffer and then incubated with p65 antibody (dilution $1: 1000$ ) for $1 \mathrm{~h}$ at $20^{\circ} \mathrm{C}$. After three successive washings, the wells were incubated for $1 \mathrm{~h}$ with dilute horseradish peroxidase-conjugated antibody (dilution 1:1000) followed by the addition of $100 \mu \mathrm{L}$ of developing solution. After 5 min of incubation, the reaction was blocked by adding $100 \mu \mathrm{L}$ of stop solution reagent. Optical density was determined using spectrophotometer at $450 \mathrm{~nm}$ with a reference wavelength of $655 \mathrm{~nm}$.

\section{Statistical analysis}

Data were analyzed using Student's $t$ test, one-way analysis of variance or Newman-Keuls multiple comparison test. If not otherwise stated, data are expressed as means \pm SD from three independent experiments. Differences were considered significant when $p<0.01$.

\section{Results}

The cytotoxic effects of AA and its analogue, THAA, were evaluated in the presence or absence of LPS $(1 \mu \mathrm{g} / \mathrm{mL})$, and neither AA nor THAA reduced the cell viability of RAW264.7 macrophages in the absence of LPS even at a dose of $80 \mu \mathrm{M}$ after a period of $24 \mathrm{~h}$ (Fig. 2). Interestingly, both AA and THAA blocked the cell death induced by LPS stimulation; the cytoprotective effects of the compounds were most significantly observed at $40 \mu \mathrm{M}$ of each compound (Fig. 2). At non-cytotoxic concentrations (5-40 $\mu \mathrm{M})$, both AA and THAA markedly reduced LPS-induced NO production in a dose-dependent manner (Fig. 3A). To examine whether AA and THAA could also inhibit PGE2 production, RAW264.7 macrophages were pre-incubated with various concentrations of AA or THAA for $1 \mathrm{~h}$, and then activated with LPS ( $1 \mu \mathrm{g} / \mathrm{mL})$ for $24 \mathrm{~h}$. As shown in Fig. 3B, both AA and THAA significantly inhibited the production of $\mathrm{PGE}_{2}$ in a dose-dependent manner. We next determined whether the inhibitory effects of AA and THAA on the production of NO 
A

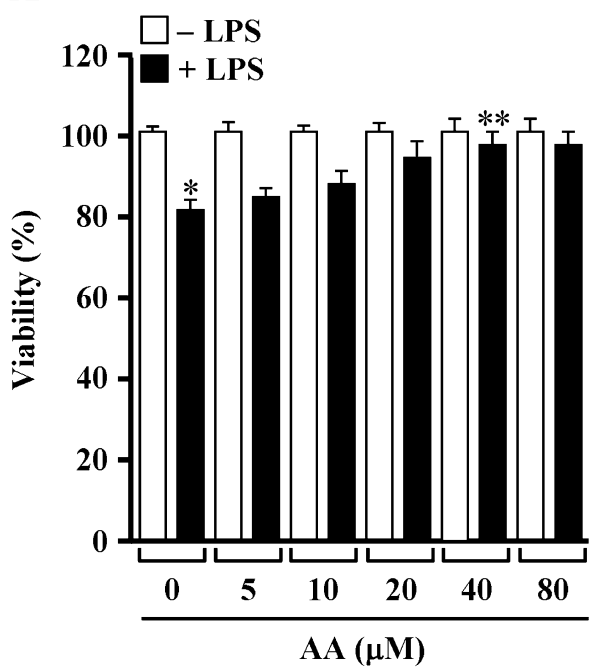

B

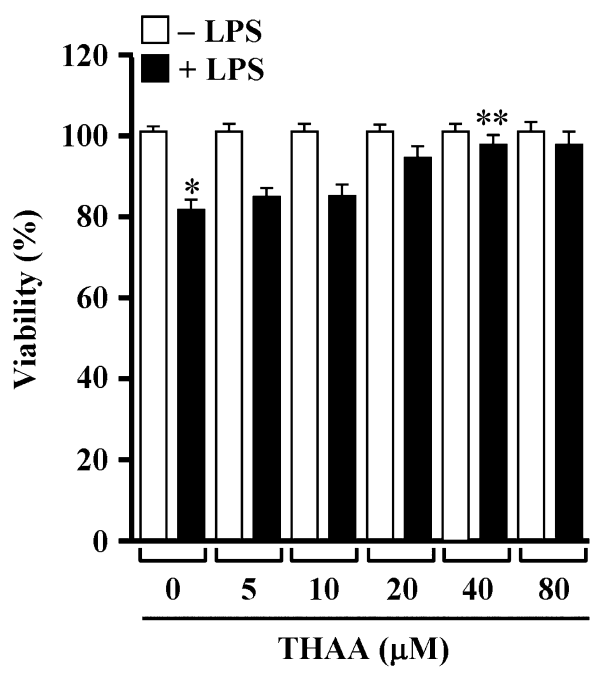

Fig. 2. Effects of AA and THAA on the cell viability of RAW264.7 macrophages. Cells were incubated with indicated concentrations of either AA (A) or THAA (B) in the absence or presence of $1 \mu \mathrm{g} / \mathrm{mL}$ of LPS for $24 \mathrm{~h}$. The cell viability was determined by MTT assay, as described in Materials and Methods. ${ }^{*} p<0.05$ as compared with the control group (cultured in medium alone). ${ }^{* *} p<0.05$ as compared with only LPS groups.

A

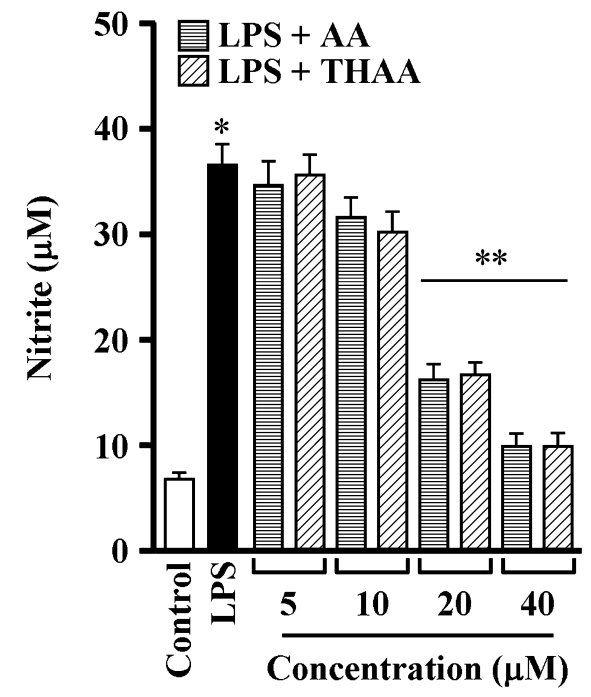

B

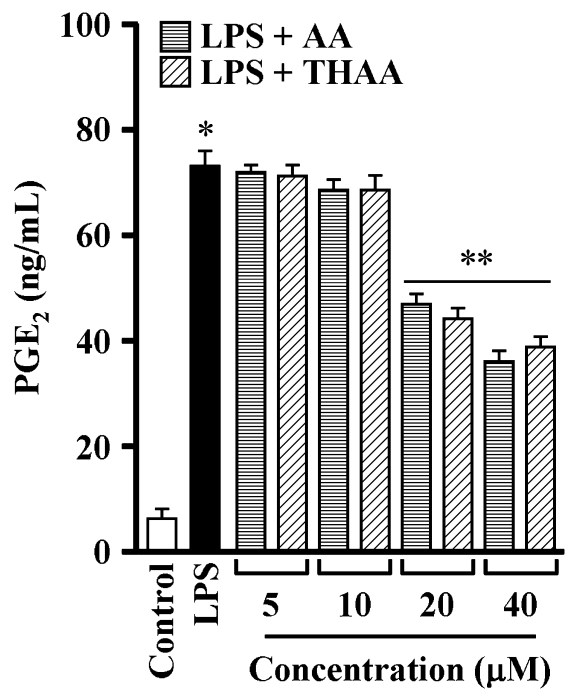

Fig. 3. Effects of AA and THAA on the production of NO and $\mathrm{PGE}_{2}$ in LPS-activated RAW264.7 macrophages. Cells were pretreated with indicated concentrations of AA or THAA for $1 \mathrm{~h}$ prior to the addition of $1 \mu \mathrm{g} / \mathrm{mL}$ of LPS, and the cells were further incubated for $24 \mathrm{~h}$. The concentrations of nitrite (A) and $\mathrm{PGE}_{2}(\mathrm{~B})$ in culture medium were determined by Griess reaction and $\mathrm{PGE}_{2}$ ELISA kit, respectively, as described in Materials and Methods. ${ }^{*} p<0.05$ as compared with the control group (cultured in medium alone). ${ }^{* *} p<0.05$ as compared with only LPS groups.

and $\mathrm{PGE}_{2}$ could be related to the modulation of the expression of the iNOS and COX-2 enzymes. In unstimulated RAW264.7 macrophages, neither iNOS nor COX-2 protein was detectable. In response to LPS, the expression levels of iNOS and COX-2 were markedly augmented, and AA significantly inhibited iNOS and COX-2 expression in a dose-dependent manner (Fig. 4A). Similar results were also observed when the effects of THAA on the LPS-induced iNOS and COX-2 expression were examined (Fig. 4B). In general, these results are consistent with the profile of the inhibitory effects of AA and THAA on $\mathrm{NO}$ and $\mathrm{PGE}_{2}$ production. We further investigated the effects of AA and 
A

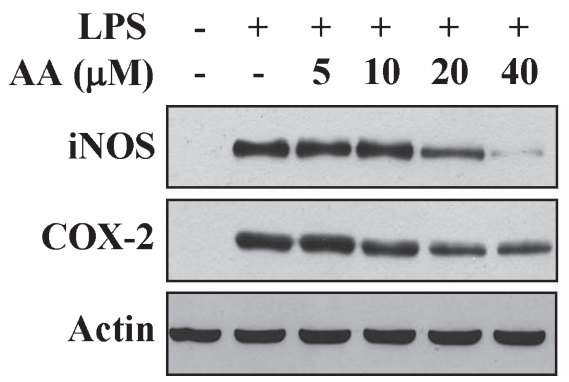

B

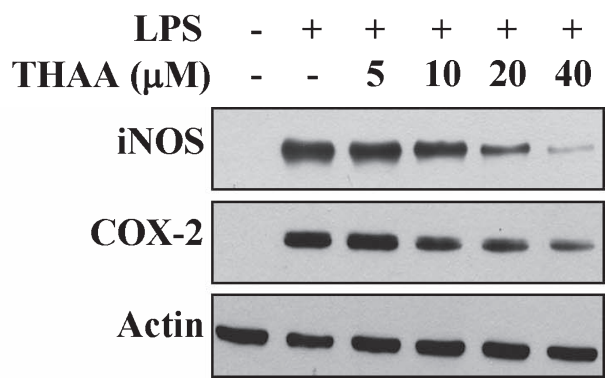

Fig. 4. Effects of AA and THAA on the expression of iNOS and COX-2 in LPS-activated RAW264.7 macrophages. Cells were pretreated with indicated concentrations of AA (A) or THAA (B) for $1 \mathrm{~h}$ prior to the addition of $1 \mu \mathrm{g} / \mathrm{mL}$ of LPS, and the cells were further incubated for $6 \mathrm{~h}$. The levels of iNOS and COX-2 proteins were monitored by Western blot analysis as described in Materials and Methods. These experiments have been repeated three times with similar observation.

A

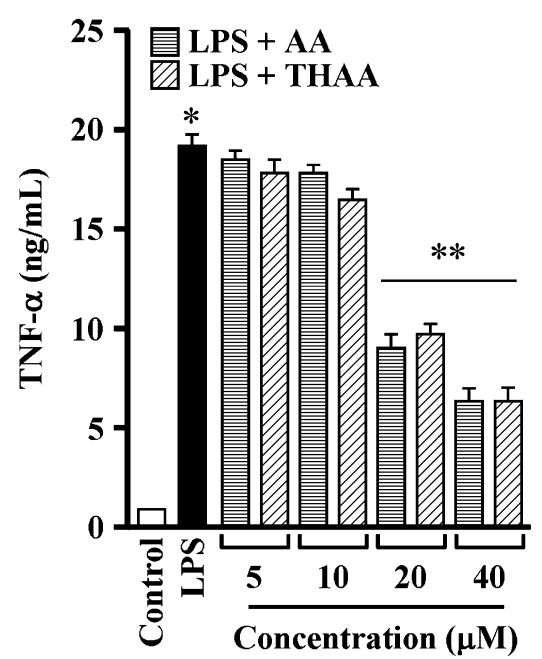

B

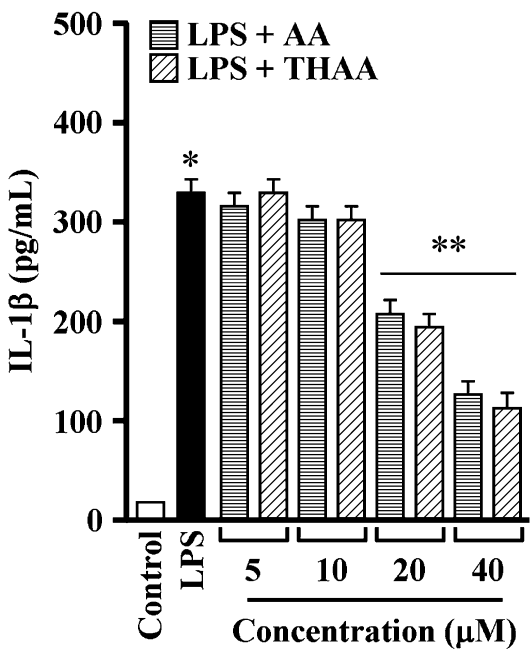

C

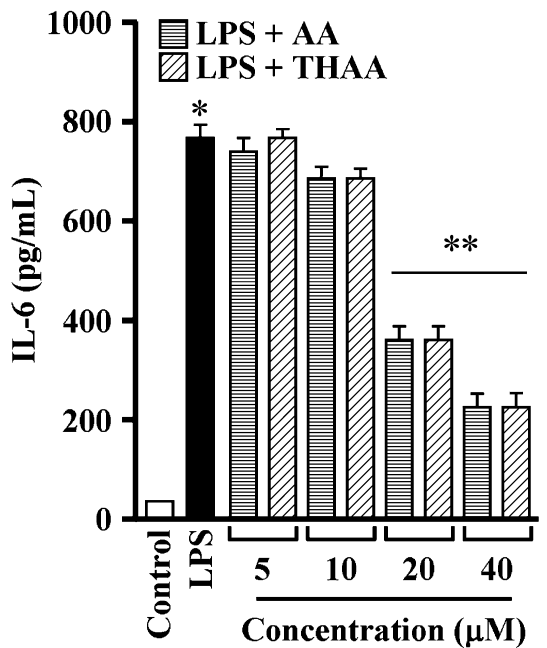

Fig. 5. Effects of AA and THAA on the production of TNF- $\alpha$, IL-1 $\beta$, and IL-6 in LPS-activated RAW264.7 macrophages. Cells were pretreated with indicated concentrations of AA or THAA for $1 \mathrm{~h}$ prior to the addition of $1 \mu \mathrm{g} / \mathrm{mL}$ of LPS, and the cells were further incubated for $24 \mathrm{~h}$. The concentrations of TNF- $\alpha$ (A), IL-1 $\beta$ (B), and IL-6 (C) in culture medium were determined by their specific antibody-coated ELISA kits as described in Materials and Methods. ${ }^{*} p<0.05$ as compared with the control group (cultured in medium alone). ${ }^{* *} p<0.05$ as compared with only LPS groups.

THAA on the LPS-induced production of TNF- $\alpha$, IL-1 $\beta$, and IL- 6 by using an enzyme immunoassay. Pre-treatment of RAW264.7 macrophages with AA or THAA for $1 \mathrm{~h}$ decreased the production of these pro-inflammatory cytokines in a dose-dependent manner (Fig. 5A, B and C). Finally, we investigated whether AA and THAA could prevent the translocation of the subunit of NF- $\mathrm{\kappa B}$, p65, from the cytosol to the nucleus after its release from its inhibitory proteins. In the case of the treatment with $40 \mu \mathrm{M}$ of AA, a decrease in the level of p65 in the nuclear fraction was detected by Western blot analysis (Fig. 6A). This cascade was also confirmed by the inhibition of the NF- $\mathrm{BB} / \mathrm{p} 65$ DNA binding activity after $2 \mathrm{~h}$ treatment with AA (Fig. 6B).
Similarly, THAA $(40 \mu \mathrm{M})$ also prevented the translocation of NF- $\mathrm{KB} / \mathrm{p} 65$ from the cytosol to the nucleus (Fig. 6A and $\mathrm{B})$. The IKB kinase (IKK) pathway was examined as another potential regulator of NF- $\mathrm{KB}$. Both AA and THAA inhibited I $\mathrm{B} \alpha$ degradation resulting from LPS stimulation (Fig. 6C).

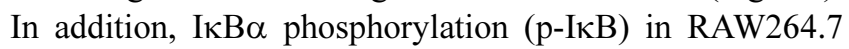
macrophages increased after LPS treatment, and this phosphorylation was also inhibited by AA or THAA (Fig. 6C).

\section{Discussion}

It has been reported that AA has anti-inflammatory effects $[11,12]$. Unfortunately, the conjugated double bonds of AA 
A

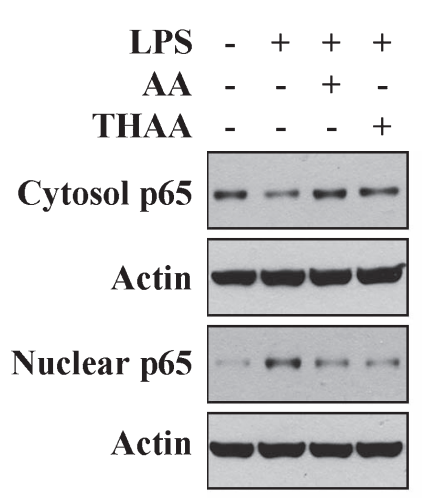

B

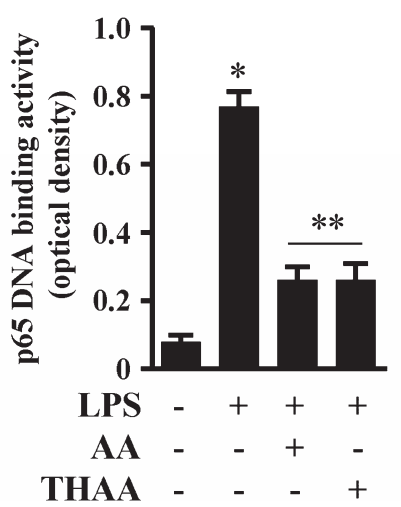

$\mathbf{C}$

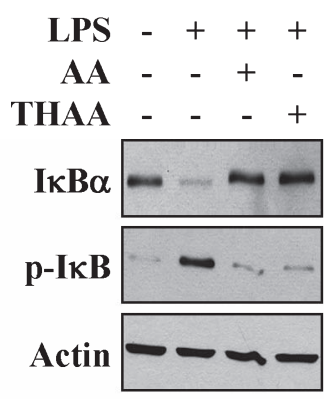

Fig. 6. Effects of AA and THAA on the nuclear translocation of NF-kB/p65 in LPS-activated RAW264.7 macrophages. Cells were treated with $40 \mu \mathrm{M}$ of either AA or THAA for $1 \mathrm{~h}$ prior to the addition of $1 \mu \mathrm{g} / \mathrm{mL}$ of LPS, and the cells were further incubated

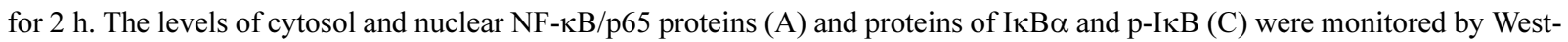
ern blot analysis as described in Materials and Methods. These experiments have been repeated three times with similar observation. The NF-kB/p65 DNA-binding activities (B) were measured by NF- $\mathrm{kB} / \mathrm{p} 65$ transcription factor assay kit as described in Materials and Methods. ${ }^{*} p<0.05$ as compared with the control group (cultured in medium alone). ${ }^{* *} p<0.05$ as compared with only LPS groups.

are readily oxidized by air and its oxidation products are considered to be responsible for the allergenic effect [9]. THAA, a modified AA analogue that is reduced by hydrogenation of two double bonds, is much more resistant to air oxidation than AA [10]. Whether THAA could exert antiinflammatory effects to the same extent as AA, however, has not been investigated so far. The present study demonstrates, for the first time, that THAA can suppress the expression of pro-inflammatory enzymes (iNOS and COX-2) and inhibit the production of pro-inflammatory cytokines (IL-1 $\beta$, IL-6, and TNF- $\alpha$ ) through inhibition of NF- $\kappa B$ activation in LPSactivated RAW264.7 macrophages.

NO, PGE2, and pro-inflammatory cytokines (e.g., IL-1 $\beta$, IL-6, and TNF- $\alpha$ ) have been implicated as important inflammatory mediators in the process of macrophage-mediated inflammation $[13,14]$. However, the uncontrolled/excess production of these mediators by activated macrophages may contribute to the development of various inflammatory diseases [15]. Hence, the inhibition of these mediators by pharmacological modulators may be an effective therapeutic strategy for preventing inflammatory reaction and diseases [16]. The present study demonstrates that THAA at noncytotoxic doses inhibited the production of $\mathrm{NO}$ and $\mathrm{PGE}_{2}$ in LPS-activated RAW264.7 macrophages. To search for the underlying mechanism of this potent effect of THAA, we examined its effects on the protein expression levels of both iNOS and COX-2 enzymes, which are responsible for $\mathrm{NO}$ and $\mathrm{PGE}_{2}$ production. We found that THAA suppressed LPS-induced iNOS and COX-2 expression, suggesting that the inhibition of $\mathrm{NO}$ and $\mathrm{PGE}_{2}$ production by THAA is a result of the inhibition of iNOS and COX-2 expression. In other experimental sets, we also examined whether THAA could inhibit the production of pro-inflammatory cytokines in LPS-stimulated RAW264.7 macrophages. Similar to its inhibitory effects on the expression of pro-inflammatory enzymes, THAA inhibited the production of TNF- $\alpha$, IL- $1 \beta$, and IL-6. Thus, our results suggest that THAA, at least in LPS-activated macrophages, may exert the anti-inflammatory effects not only by suppressing the expression of proinflammatory enzymes but also by inhibiting the production of pro-inflammatory cytokines.

In our study, we also performed a comparative study of the anti-inflammatory effects of AA and THAA at the same concentration, and found that THAA had almost the same inhibitory effects as AA on the production of proinflammatory mediators in LPS-activated RAW264.7 macrophages, indicating that the presence of the conjugated double bonds of AA is not essential for anti-inflammatory activity; i.e., the hydrogenation of these bonds results in no significant effect on the anti-inflammatory activity of AA. Moreover, both THAA and AA effectively blocked the activity of $\mathrm{NF}-\kappa \mathrm{B}$, a key transcription factor that can induce the expression of pro-inflammatory enzymes and cytokines, and their inhibitory effects on NF-kB activation were almost identical, suggesting that both THAA and AA may inhibit the production of the inflammatory mediators, presumably through the mechanism(s) associated with NF- $\mathrm{kB}$ inactivation. Thus, our results confirm that THAA, a reduced AA analogue with higher oxidative stability than AA [10], retains the antiinflammatory activity of its parent compound, AA.

Under normal conditions, NF- $\mathrm{KB}$ associates with inhibitory $\mathrm{I} \kappa \mathrm{B}$ proteins which sequester NF- $\mathrm{KB}$ in the cytoplasm. 


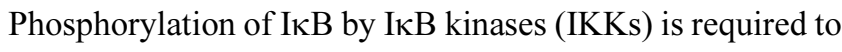
release NF- $\kappa B$ from this inhibition [4]. Indeed, LPS stimulation of RAW264.7 macrophages activates several intracellular signaling pathways, including the IкB kinase (IKK)/ NF- $\kappa B$ pathway [17]. Our results suggest that both AA and THAA impinge on the IKK pathway to dampen the inflammatory response by repressing IKB phosphorylation and subsequent NF- $\mathrm{KB}$ activation (Fig. 6). Accordingly, the inhibition of I $\mathrm{B}$ phosphorylation and degradation by AA or THAA during LPS-induced inflammation may limit NF- $\mathrm{KB}$ activation.

\section{Conclusion}

The data presented in this study lead to the following major conclusions: (i) in addition to AA, THAA, a reduced analogue of $\mathrm{AA}$, is also able to inhibit the production of $\mathrm{NO}$ and $\mathrm{PGE}_{2}$ by suppressing the expression of iNOS and COX-2 as well as the production of pro-inflammatory cytokines (IL-1 $\beta$, IL-6, and TNF- $\alpha$ ) in LPS-activated RAW264.7 macrophages; (ii) both THAA and AA prevent the nuclear translocation of $\mathrm{NF}-\mathrm{\kappa B} / \mathrm{p} 65$, presumably suggesting that THAA may exert anti-inflammatory effects through the same mechanism as AA; (iii) the anti-inflammatory effects of THAA and AA are almost identical, indicating that THAA retains the anti-inflammatory activity of AA.

\section{Acknowledgments}

This work was supported by the Korea Research Foundation Grant funded by the Korean Government (MOEHRD) (KRF-2006-005-J03202).

\section{References}

[1] Hunter, M., Wang, Y., Eubank, T., Baran, C., Nana-Sinkam, P., and Marsh, C.: Survival of monocytes and macrophages and their role in health and disease. Front Biosci., 14, 40794102, 2009.

[2] Murakami, A. and Ohigashi, H.: Targeting NOX, iNOS and COX-2 in inflammatory cells: chemoprevention using food phytochemicals. Int. J. Cancer, 121, 2357-2363, 2007.

[3] Hawiger, J.: Innate immunity and inflammation: a transcriptional paradigm. Immunol. Res., 23, 99-109, 2001.

[4] Magnani, M., Crinelli, R., Bianchi, M., and Antonelli, A.: The ubiquitin-dependent proteolytic system and other potential targets for the modulation of nuclear factor- $\kappa \mathrm{B}(\mathrm{NF}-\kappa \mathrm{B})$. Curr. Drug Targets, 1, 387-399, 2000.
[5] Guha, M. and Mackman, N.: LPS induction of gene expression in human monocytes. Cell Signal., 13, 85-94, 2001.

[6] Surh, Y.J., Kundu, J.K., Na, H.K., and Lee, J.S.: Redoxsensitive transcription factors as prime targets for chemoprevention with anti-inflammatory and antioxidative phytochemicals. J. Nutr., 135, 2993S-3001S, 2005.

[7] Craig, W.J.: Health-promoting properties of common herbs. Am. J. Clin. Nutr., 70, 491S-499S, 1999.

[8] Funk, C. and Croteau, R.: Diterpenoid resin acid biosynthesis in conifers: characterization of two cytochrome P450dependent monooxygenases and an aldehyde dehydrogenase involved in abietic acid biosynthesis. Arch. Biochem. Biophys., 308, 258-266, 1994.

[9] Sadhra, S., Foulds, I.S., and Gray, C.N.: Identification of contact allergens in unmodified rosin using a combination of patch testing and analytical chemistry techniques. $\mathrm{Br}$. J. Dermatol., 134, 662-668, 1996.

[10] Karlberg, A.T., Boman, A., and Nilsson, J.L.: Hydrogenation reduces the allergenicity of colophony (rosin). Contact Dermatitis, 19, 22-29, 1988.

[11] Fernández, M.A., Tornos, M.P., García, M.D., de las Heras, B., Villar, A.M., and Sáenz, M.T.: Anti-inflammatory activity of abietic acid, a diterpene isolated from Pimenta racemosa var. grissea. J. Pharm. Pharmacol., 53, 867-872, 2001.

[12] Takahashi, N., Kawada, T., Goto, T., Kim, C.S., Taimatsu, A., Egawa, K., Yamamoto, T., Jisaka, M., Nishimura, K., Yokota, K., Yu, R., and Fushiki, T.: Abietic acid activates peroxisome proliferator-activated receptor- $\gamma(\operatorname{PPAR} \gamma)$ in RAW264.7 macrophages and 3T3-L1 adipocytes to regulate gene expression involved in inflammation and lipid metabolism. FEBS Lett., 550, 190-194, 2003.

[13] Cho, H.J., Seon, M.R., Lee, Y.M., Kim, J., Kim, J.K., Kim, S.G., and Park, J.H.: 3,3'-Diindolylmethane suppresses the inflammatory response to lipopolysaccharide in murine macrophages. J. Nutr., 138, 17-23, 2008.

[14] Wu, D. and Meydani, S.N.: Age-associated changes in immune and inflammatory responses: impact of vitamin $\mathrm{E}$ intervention. J. Leukoc. Biol., 84, 900-914, 2008.

[15] Glaros, T., Larsen, M., and Li, L.: Macrophages and fibroblasts during inflammation, tissue damage and organ injury. Front Biosci., 14, 3988-3993, 2009.

[16] Xia, Z. and Triffitt, J.T.: A review on macrophage responses to biomaterials. Biomed. Mater., 1, R1-R9, 2006.

[17] Rajapakse, N., Kim, M.M., Mendis, E., and Kim, S.K.: Inhibition of inducible nitric oxide synthase and cyclooxygenase2 in lipopolysaccharide-stimulated RAW264.7 cells by carboxybutyrylated glucosamine takes place via downregulation of mitogen-activated protein kinase-mediated nuclear factor- $\mathrm{kB}$ signaling. Immunology, 123, 348-357, 2008. 